\title{
Medical Imaging, Virtual Reality
}

National Cancer Institute

\section{Source}

National Cancer Institute. Medical Imaging, Virtual Reality. NCI Thesaurus. Code C18869.

Virtual reality in Medical Imaging is the technique of combining 3D DICOM image data sets produced by medical imaging equipment with extremely high-powered computer capabilities. Virtual reality employs medical images as a foundation to create innovative methods of reviewing and analyzing the data they produce. 